\title{
HIPERTENSÃO ARTERIAL SISTÊMICA NA ATENÇÃO BÁSICA DE SAÚDE
}

\author{
SYSTEMIC ARTERIAL HYPERTENSION IN BASIC HEALTH CARE
}

\author{
Natalle da Conceição Ramos ${ }^{1}$ \\ Jessica Alves Moreira ${ }^{2}$ \\ Gislayne Tacyana dos Santos Lucena ${ }^{3}$ \\ Maria Iranilda Silva Magalhães ${ }^{4}$
}

RESUMO: Objetivo: Analisar a prestação de serviço na Atenção Básica (AB) para os diagnósticos de Hipertensão Arterial Sistêmica (HAS) e/ou Diabetes mellitus (DM) aos usuários do Sistema Único de Saúde (SUS) a partir das políticas públicas. Metodologia: O estudo foi do tipo transversal descritivo e quantitativo, possibilitando acesso documental a partir dos prontuários e registros de hipertensos(as) e/ou diabéticos(as), correlacionando os fatos sem manipulação dos dados obtidos. A pesquisa foi realizada na Unidade Básica de Saúde (UBS) Maria José de Jesus, situada na Rua Coronel Guimarães, número 157, Bairro Centro, no município de Cajazeiras, estado da Paraíba. Resultados: Constatou-se mediante os dados que dentre a população afetada pela HAS houve uma maior prevalência sobre o sexo feminino, quando comparado ao masculino e a faixa etária mais afetada foram àqueles com idade igual ou superior a 65 anos, e que a maioria dos dados obtidos nos prontuários aponta um percetual de pressão arterial com níveis de 130x80 $\mathrm{mmHg}$, o qual corresponde a $18,18 \%$, já o DM esteve presente em $52,27 \%$ dos pesquisados. Conclusão: Os registros públicos na $A B$ de saúde no diagnóstico de Hipertensão Arterial Sistêmica e Diabetes Mellitus possuem informação insuficiente nos portuários obtidos na pesquisa. Entretanto, os protocolos da HAS e DM são desejáveis pela Educação em Saúde dentro das políticas que tentam promover a prevenção das doenças, promoção da saúde e bem-estar.

\footnotetext{
${ }^{1}$ Acadêmica do Curso de Bacharelo em Biomedicina da Faculdade Santa Maria (FSM), CajazeirasPB. E-mail: natallecramos59@gmail.com.

2 Especialista em Hematologia Clínica pelo Institulo Leão Sampaio Ensino Universitário. Biomédica formada pelo Instituto Leão Sampaio Ensino Universitário. Especialista em Docência do Ensino Superior pela Faculdade Santa Maria - FSM. Docente do Curso de Bacharelado em Biomedicina da Faculdade Santa Maria (FSM), Cajazeiras-PB. E-mail:jessica.alvesmoreira@hotmail.com.

${ }_{3}^{3}$ Metre em Ciência Animal pelo Universidade Federal de Campina Grande, Campus de Patos - PB. Biomédica formada pela Faculdade de Integradas de Patos (2014). Docente do curso de Bacharelado em Biomedicina da Faculdade Santa Maria (FSM), Cajazeiras-PB.

${ }^{4}$ Doutorando do Programa de Pós Graduação de Pesquisa e Inovação da Faculdade de Medicina do ABC Paulista. Mestre em Ciências de Saúde pela Universidade Cruzeiro do Sul. Especialista em Ciências da Educação, Biologia e Psicopedagogia. Biomédica formada pelo Instituto Leão Sampaio Ensino Universitário. E-mail: iranildamagalhaes@gmail.com.
} 
Palavras chave: Educação em Saúde; Hipertensão Arterial Sistêmica; Políticas Públicas.

ABSTRACT: Objective: To analyze the provision of services in Basic Health Care (BHC) for the diagnosis of Systemic Arterial Hypertension (SAH) and/or Diabetes mellitus (DM) to the users of the Unified Health System (UHS) based on public policies. Methodology: This was a descriptive, quantitative and cross-sectional study, allowing documentary access from the records of hypertensive and/or diabetic patients, correlating the facts without manipulating the data obtained. The study was conducted at the Maria José de Jesus Basic Health Unit (BHU), located at Rua Coronel Guimarães,157, Centro, in the city of Cajazeiras, state of Paraíba. Results: We found that among the population affected by $\mathrm{SH}$ there was a greater prevalence in females, when compared to males and the age group most affected were those aged 65 or over, and that most data obtained in the protuaries shows a percetual blood pressure with levels of $130 \times 80 \mathrm{mmHg}$, which corresponds to $18.18 \%$, and diabetes mellitus was present in $52.27 \%$ of those surveyed. Conclusion: The public health programs in the diagnosis of Systemic Arterial Hypertension and Diabetes Mellitus have insufficient information based on the records obtained in the research. However, the protocols for SAH and DM are desirable for Health Education within policies that seek to promote disease prevention, health promotion and well-being.

Keywords: Health Education; Systemic Arterial Hypertension; Public Policies. 


\section{INTRODUÇÃO}

A HAS é um grave problema de Saúde Pública no Brasil e no mundo, contando com uma incidência no Brasil que varia de $22 \%$ para adultos, chegando a mais de $50 \%$ para pessoas com 60 a 69 anos e $75 \%$ em pessoas com mais de 70 anos de idade (SBC, 2010). É um mecanismo que altera a pressão dos vasos sanguíneos, configurando-se como principal fator de risco e, na maioria das vezes, conjugada com a DM, ampliando, portanto, os riscos cardiovasculares reversíveis ou irreversíveis (TORTORELLA, 2017).

Estudos apontam que as pessoas acometidas pela obesidade configuram em $20 \%$ a $30 \%$ da população, constando uma prevalência principalmente acima de 60 anos.Contudo, a utilização de fármaco e a prática de atividade anti-hipertensiva diminuem $13 \%$ da mortalidade, e $30 \%$ de ser acometido(a) pelas consequências da doença, assim como o Acidente Vascular Cerebral (AVC) e $23 \%$ e de patologias coronarianas (LONGO, M.A.T.; MARTELLI, A,; ZIMMERMANN, A. 2011).

Nesse contexto, Mendes (2011) relata que a HAS atinge aproximadamente $20 \%$ da população adulta brasileira e a expectativa para a DM é que, em 2025, alcance o número de 350 milhões de pessoas acometidas por esta doença. O SUS, no nível da $A B$, propicia aos usuários ações educativas para o diagnóstico e acompanhamento e intervenção as pessoas com HAS e/ou DM, favorecendo a promoção, proteção e recuperação do bem-estar físico, psicológico e psíquico. Essas atividades conjugaram-se na perspectiva de consolidar o protocolo do cuidado as doenças crônicas, propiciando qualidade de vida e bem-estar social para os usuários do SUS (MINISTÉRIO DA SAÚDE, 2014).

A Política Nacional da Atenção Básica (PNAB), com o avanço do SUS e a intervenção da Sociedade Brasileira de Cardiologia (SBC), bem como as Políticas Públicas do Conselho Nacional de Saúde e Setoriais, favorecem orientações sob protocolos e diretrizes para que os trabalhadores e gestores do sistema possam 
minimizar a prevalência da HAS e/ou DM na sociedade por meio da Educação em Saúde (TAVARES, 2010).

Existem meios de controle e equilíbrio para HAS e/ou DM, uma vez que acompanham o nível de melhora e amenizam o risco de morbidade e óbito. Isso acontece por serem enfermidades crônicas que necessitam de auxílio e de recursos terapêuticos e farmacológicas. (BURGOS, Miria Suzana et al. 2015).

A educação em saúde tem um papel fundamental de discussão, identificação, sensibilização para prevenir doenças e promover saúde, assim como a expectativa do trabalho com profissionais multidisciplinares seja capaz de desenvolver na $A B$ atividades educacionais, subsidiando benefícios que auxiliem hipertensos(as) a obterem hábitos de vida saudável (RADOVANOVIC et al., 2016).

De acordo com o Ministério de Saúde (2006), 42,7\% dos cidadãos brasileiros têm sido afetados por enfermidades crônicas. Esses dados alertam as Secretarias dos Estados e Municípios para aplicação de programas educacionais nas unidades básicas do SUS a fim de promover a sensibilização aos usuários para práticas de hábitos que favoreçam qualidade de vida saudável e reduzam os índices de incidência das Doenças Crônicas Não Transmissíveis (DCNT), nesse estudo, a Hipertensão Arterial Sistêmica (HAS) e/ou Diabetes Mellitus (DM) (MACHADO et al., 2016).

\section{MATERIAIS E MÉTODOS}

A pesquisa quantitativa foi uma coleta sistemática de informações, onde o pesquisador reúne, registra e analisa os dados, utilizando técnicas estatísticas, desde que mantenha segurança e sigilo dos dados obtidos.

O estudo será realizado no município de Cajazeiras, estado da Paraíba, na Unidade Básica de Saúde (UBS) Maria José de Jesus, situada na Rua Coronel Guimarães, número 157, Bairro Centro. O município de Cajazeiras encontra-se no sertão paraibano, no extremo oeste do estado da Paraíba, região Nordeste do país, a cerca de 460 quilômetros de distância da capital do estado, João Pessoa. De 
acordo com o Instituto Brasileiro de Geografia e Estatística (IBGE, 2017), o município de Cajazeiras possui uma população aproximada de 62.187 habitantes, correspondendo ao oitavo município mais populoso da Paraíba (IBGE, 2017).

Projetou-se analisar 100 prontuários de homens e mulheres cadastrados na Unidade Básica de Saúde Maria José de Jesus com diagnóstico de Hipertensão Arterial Sistêmica (HAS). Entretanto, somente 44 (quarenta e quatro) prontuários foram habilitados para o estudo.

Inicialmente, foi enviado um ofício à coordenação, apresentando os objetivos da pesquisa e solicitando a autorização para realizá-la, por meio da emissão do Termo de Anuência e Assinatura do Termo de Consentimento para Uso de Dados TCUD. Para realizar a coleta de dados, o presente estudo foi submetido à apreciação e aprovação pelo Comitê de Ética em Pesquisa da Faculdade Santa Maria (CEP-FSM). Logo após a aprovação pela referida instância colegiada, os dados foram coletados.

Ao cumprir o protocolo prévio de iniciação à pesquisa, foi encaminhadaà instituição coparticipante a aprovação da pesquisa pelo Comitê de Ética e Pesquisa (CEP) para a operacionalização do processo da apropriação dos dados. Na ocasião, o pesquisador apresenta a sugestão do cronograma de trabalho.

Os dados utilizados para a pesquisa foram tratados com total responsabilidade e sigilo. Os mesmos foram coletados entre os meses de abril e maio de 2019 através de um instrumento próprio, elaborado pelos pesquisadores.

Esta pesquisa foi realizada na Unidade Básica de Saúde (UBS) Maria José de Jesus, no município de Cajazeiras, estado da Paraíba, por meio da análise documental. Os dados foram extraídos de prontuários de usuários diagnosticados com Hipertensão Arterial Sistêmica (HAS). Foi elaborado um formulário próprio para a coleta de dados presente no ANEXO E, e de acordo com os dados estatísticos recolhidos, serão guardados para que possam facilitar uma posterior análise.

Todos os dados coletados através do formulário foram analisados estatisticamente e armazenados. Foram organizados com registros no programa Statistical Productand Service; Solutions (SPSS) versão 25 e Microsoft Office Excel versão 2016. Em seguida, os dados foram analisados empregando a estatística descritiva, por meio de cálculos de frequência absoluta e relativa (\%), medidas de 
tendência central e dispersão (média, mediana e desvio-padrão) conforme o tipo de variável. Para comparação de diferenças ou relação entre variáveis, utilizou-se estatística inferencial conforme o tipo de variável e distribuição apresentada, considerando nível de confiança de $95 \%$. Os resultados foram discutidos à luz da literatura pertinente.

\section{RESULTADOS E DISCUSSÕES}

A pesquisa foi realizada na Unidade Básica de Saúde (UBS) Maria José de Jesus, no município de Cajazeiras, estado da Paraíba, situada à rua Coronel Guimarães, número 157, bairro Centro. A coleta utilizou dados secundários organizados sob registro em prontuários e alimentados pela equipe da Atenção Básica de Saúde (ABS). O foco do estudo pautou-se nos usuários do SUS diagnosticados com Hipertensão Arterial Sistêmica (HAS) e/ou Diabetes Mellitus (DM). No projeto de estudo, configurava-se uma expectativa de 100 (cem) amostras, entretanto, baseado no critério de inclusão somente 44 (quarenta quatro) prontuários estavam aptos a participar da pesquisa. 
Tabela 1. Perfil dos usuários do SUS com os diagnósticos de HAS e/ou DM ( $n=44)$.

\begin{tabular}{lcc}
\hline \multicolumn{1}{c}{ Variáveis } & fabsoluta & f relativa (\%) \\
\hline Gênero & & \\
Feminino & 25 & $56,81 \%$ \\
Masculino & 19 & $43,19 \%$ \\
Faixa etária (anos) & & \\
$40-64$ & 10 & $22,72 \%$ \\
$65-83$ & 64 & $77,28 \%$ \\
Média \pm DP & $68,81 \%$ \\
Pressão arterial (mmHg) & 20 & $66,81 \%$ \\
100x80 à 120x80 & 24 & $47,73 \%$ \\
130x80 à 190x60 & $130 \times 80$ & $52,27 \%$ \\
Média & & $18,18 \%$ \\
DM & 23 & $52,27 \%$ \\
Sim & 21 & $47,73 \%$ \\
Não & & \\
Medidas & 44 & $100,00 \%$ \\
Sim & 0 & $00,00 \%$ \\
Não & & \\
Exames & 43 & $97,72 \%$ \\
Sim & 1 & $02,28 \%$ \\
Não & & \\
\hline
\end{tabular}

Quanto ao gênero, $56,81 \%$ da amostra da pesquisa eram do sexo feminino e os $43,19 \%$ eram do sexo masculino, demostrando que o sexo feminino prevaleceu como maioria para o diagnóstico de HAS em relação ao sexo masculino 43,19\%. Segundo Silva (2016), a predominância de mulheres hipertensas relaciona-se a diversos fatos, exemplificado o uso de contraceptivos, síndrome do ovário policístico, gestação, reposição hormonal e menopausa, elevando o nível da pressão arterial (SILVA, 2016).

$\mathrm{Na}$ análise sobre a faixa etária, constata-se, na amostra, menor idade de registro completo de 40 anos, com esse dado, verificou-se que, no grupo etário de 40 a 64 anos de idade $(22,72 \%)$, o nível da pressão arterial tende a elevar-se, causando HAS. Porém, a prevalência configura-se na faixa etária de 65 a 83 anos de idade (77,28\%). Conforme Malta (2017), a prevalência associada à HAS está relacionada à idade igual ou maior a 18 anos, com predominância para o sexo feminino, contudo, esse número exacerba a partir dos 65 anos de idade. 
O estudo demonstrou, conforme os dados coletados, que existe um controle na pressão arterial, variando de 100x80 a 120x80 mmhg (47,73 \%), embora existam hipertensos com um desequilíbrio de 130x80 mmhg, chegando a 190x60 mmh. De acordo com o Ferreira (2009), estudos afirmam que, quando a pressão arterial sistólica é de $140 \mathrm{nmHg}$ e a diastólica de $90 \mathrm{nmHg}$, existe um grande risco para doenças cardiovasculares, alémde levar a uma hipertensão leve. Paciente acima de 18 anos, com PAS de 120 nmhg e PAD de 80 nmhg possui menor risco de patologias cardiovasculares, embora o limite aceitável seja PAS de 130 a 139 nmhg e PAD de 85-89 nmhg.

Almeida (2014) aponta que as Unidades Básicas de Saúde estão estruturadas com equipe multidisciplinar, possibilitando práticas educativas que favorecem o bemestar dos usuários do SUS com o diagnóstico de HAS por meio de estratégias e práticas de educação em saúde que permitem uma melhoria na qualidade de vida. (ALMEIDA. MOUTINHO, LEITE, 2014).

Observou-se uma associação entre o diagnóstico de HAS e DM, alcançando $52,27 \%$ da amostra desse estudo com os dois diagnósticos simultaneamente. De acordo com Filho (2014), uma associação entre hipertensão e diabetesé comum nos registros da Atenção Básica, bem como esses fatores persistem e causam, na maioria das vezes, complicações, principalmente em relação às doenças cardiovasculares (FILHA; NOGUEIRA; MEDINA, 2014).

O estudo aponta que $100 \%$ da amostra continham o cálculo do ICM registrado e apresentavam sobrepeso (medidas). Na pesquisa de Amer (2011), ressalta-se que um número elevado de hipertensos tem sobrepeso, causando riscos das complicações dos diagnósticos de HAS e/ou DM (AMER; MARCON; SANTANA, 2011).

Nos registros, percebeu-se que um pequeno percentual $(2,28 \%)$ não estava com os exames de rotina realizados em relação aos diagnósticos de HAS e/ou DM. A Linha de Guia de Hipertensão Arterial da Secretária de Saúde do Paraná apresenta a importância dos exames complementares para a investigação do histórico clínico (SESA, 2014). 


\section{CONCLUSÃO}

Conclui-se que o Ministério da Saúde constitui programas nas políticas públicas de saúde para os diagnósticos de Hipertensão Arterial Sistêmica (HAS) e Diabetes Mellitus (DM) no Sistema Único de Saúde (SUS), com o objetivo de acompanhar a incidência e a prevalência dos referidos diagnósticos. Entretanto, na prática, especificamente na Atenção Básica, são muitos os desafios da equipe de saúde para a prestação de serviços. Constata-se a incompletude nos registros, dificultando a análise do estudo. No entanto, observa-se que a equipe de saúde e os usuários não se apoderam dos protocolos com a relevância cabível, possibilitando detectar que os programas para os diagnósticos de HAS e DM não são exequíveis no contexto real. Os prontuários foram considerados insatisfatórios para o objetivo da pesquisa, cujos registros possibilitaram detectar as fragilidades do sistema, uma vez que, na medição registrada, consta elevação na pressão arterial continuamente. Aqueles que apresentam dados nos prontuários com a medição da pressão arterial em equilíbrio, percebe-se que são assíduos às visitas. Sob a luz dos protocolos para a HAS e DM, é desejável uma postura de Educação em Saúde pautada nos parâmetros didático-pedagógicos, sistematizados por uma educação popular.Investimento, regulação e supervisão são os fatores que beneficiarão as políticas públicas para a prevenção das doenças, a promoção da saúde e o controle e bem-estar de hipertensos(as) e/ou diabéticos(a).

\section{REFERÊNCIAS BIBLIOGRÁFICAS}

ALMEIDA, Edmar Rocha; MOUTINHO, Cinara Botelho; LEITE, Maisa Tavares de Souza. A prática da educação em saúde na percepção dos usuários hipertensos e diabéticos. Saúde em debate, v. 38, p. 328-337, 2014.

AMER, Nadia Mohamed; MARCON, Sonia Silva; SANTANA, Rosangela Getirana. Índice de massa corporal e hipertensão arterial em indivíduos adultos no Centro-Oeste do Brasil. Arq Bras Cardiol, v. 96, n. 1, p. 47-53, 2011.

BURGOS, Miria Suzana et al. Interdisciplinaridade e promoção da saúde na educação básica e no sistema de saúde. 2015. 
FERREIRA, Karla de Araujo et al. Análise de custo-efetividade de procedimentos para o adiamento dafase terminal da doença renal crônica associada ao diabetes mellitus e à hipertensão arterial sob a perspectiva do Sistema Único de Saúde. 2009. Tese de Doutorado.

FILHA, Carvalho et al. Avaliação do controle de hipertensão e diabetes na Atenção Básica: perspectiva de profissionais e usuários. Saúde em Debate, v. 38, p. 265-278, 2014.

IBGE: Instituto Brasileiro de Geografia e Estatística, 2017.

LONGO, M.A.T.; MARTELLI, A,; ZIMMERMANN, A. Hipertensão Arterial Sistêmica: aspecto clínicos e análise farmacológica no tratamento dos pacientes de um setor de psicogeriatria do Instituto Bairral de Psiquiatria, no município de Itapira, SP. Revista Brasileira de Geriatria e Gerontoloia. Rio de Janeiro, v. 14, n. 2, p. 271-284, 2011.

MACHADO, A.P.et al. Estratégias educativas para prevenção de diabetes, hipertensão e obesidade. Revistada Associação Médica Brasileira. São Paulo, vol.62, n.8, p. 800808, 2016.

MALTA, Deborah Carvalho et al. Prevalência e fatores associados com hipertensão arterial autorreferida em adultos brasileiros. Rev. Saúde Pública, v. 51, n. suppl 1, p. -, 2017.

MENDES, Eugênio Vilaça. As redes de atenção à saúde. Organização Pan-Americana da Saúde, 2011.

MINISTÉRIO DA SAÚDE, Secretaria de Atenção à Saúde, Departamento de Atenção Básica Diretrizes para o cuidado das pessoas com doenças crônicas nas redes de atenção à saúde e nas linhas de cuidado prioritárias Brasília - DF 2013.

RADOVANOVIC, C.A.T. et al. Intervenção multiprofissional em adultos com hipertensão arterial: ensaio clínico randomizado. Revista Brasileira de Enfermagem. Brasília, vol. 69, n.6, p. 10671073, 2016.

SESA. Secretaria de Estado da Saúde do Paraná. Superintendência de Atenção à Saúde. Linha guia de hipertensão. - Curitiba - PA, 2014.

SILVA, Elcimary Cristina et al. Prevalência de hipertensão arterial sistêmica e fatores associados em homens e mulheres residentes em municípios da Amazônia Legal. Revista Brasileira de Epidemiologia, v. 19, p. 38-51, 2016.

Sociedade Brasileira de Cardiologia / Sociedade Brasileira de Hipertensão / Sociedade Brasileira de Nefrologia. VI Diretrizes Brasileiras de Hipertensão. Arq Bras Cardiol 2010; 95(1 supl.1): $1-51$.

TAVARES, A. et al. VI Diretrizes Brasileiras de Hipertensão. Arq Bras Cardiol 2010; 95(1 supl.1): $1-51$.

TORTORELLA, C.C.S. et al. Tendência temporal da prevanlêciade hipertensão arterial sistêmica e diabetes mellitus entre adultos cadastrados no Sistema Único de Saúde em Florianópolis, Santa Catarina, 2004-2011. Epidemiol. Serv. Saúde 26 (3) Jul-Sep 2. 